ORIGINAL PAPER

\title{
DiagnOSTIC VALUE OF TROP-2 EXPRESSION IN PAPILlaRY THYROID CARCINOMA AND COMPARISON WITH HBME-1, GALECTIN-3 AND CYTOKERATIN 19
}

\author{
Afsin Rahman Murtezaoglu*, Hasan Gucer* \\ Department of Pathology, Recep Tayyip Erdogan University, Rize, Turkey \\ ${ }^{*}$ These are co-contributing first authors of this manuscript.
}

\begin{abstract}
In this study, we compared the diagnostic value of TROP-2 expression in distinguishing between benign and malignant thyroid lesions to those of HBME-1, CK19 and galectin-3.

We selected 102 cases from our archive including 20 normal thyroid tissues, 23 follicular nodular diseases, 17 follicular adenomas, 20 follicular variant papillary carcinomas and 22 classical variant papillary carcinomas. Tissue microarrays constructed from these cases were immunohistochemically analyzed with HBME-1, CK19, galectin-3 and TROP-2.

Respectively $73.8 \%, 83.3 \%, 69 \%$ and $50 \%$ of all papillary carcinomas were positive with HBME-1, CK19, galectin-3 and TROP-2. CK19 was positive respectively by $100 \%, 43.5 \%$ and $35.3 \%$ in cases of normal thyroid, follicular nodular diseases and follicular adenoma, while the other markers were negative. In distinguishing benign and malignant lesions, which constitutes this study, HBME-1, CK19, galectin-3 and TROP-2 were statistically significant ( $\mathrm{p}<0.001)$. In distinguishing cases of follicular variant papillary carcinoma from follicular nodular diseases and follicular adenoma, HBME-1 and galectin-3 were statistically significant ( $\mathrm{p}<0.001)$.

Consequently, in this study, we found that all immunohistochemical markers were effective in distinguishing benign and malignant thyroid lesions. In determining malignancy, HBME-1 had the highest diagnostic accuracy, while CK19 was the most sensitive marker. The sensitivity increased when the markers were used together.
\end{abstract}

Key words: papillary thyroid carcinoma, TROP-2, HBME-1, galectin-3, cytokeratin19.

\section{Introduction}

The thyroid gland malignancy that pathologists most frequently enface is papillary carcinoma by 80 $85 \%$ frequency [1]. While it is generally easy to diagnose cases that show characteristic nuclear details for papillary carcinoma, there may be diagnostic conflicts even among experienced endocrine pathologists regarding cases that have follicular growth patterns [2]. Correct pathological diagnosis is necessary to minimize emotional stress, over-treatments and related financial costs brought by the diagnosis of thyroid cancer [3]. In order to eliminate disagreements among pathologists about well-differentiated lesions of thyroid derived from follicular epithelial cells and achieve diagnostic standardization, there have been many studies made where immunohistochemical and molecular markers are studied $[4,5,6,7,8,9,10,11$, 12]. The immunohistochemical markers Hector Battifora mesothelial cell epitope-1 (HBME-1), galectin 3 
(Gal-3) and cytokeratin 19 (CK19) accepted to be indicators of malignancy in thyroid lesions have been used as products of such studies as panels in most pathology laboratories. While some of the diagnostic issues are overcome by the usage of these universal markers, the search for an ideal biomarker to precisely detect malignity is still going on.

The human trophoblast cell surface antigen (TROP-2) is a transmembrane glycoprotein with a weight of $35 \mathrm{kDa}$ which is coded by the gene localized on the $1 \mathrm{p} 32$ chromosome. TROP-2, which is expressed on normal levels in various normal tissues especially placenta, is related to tumor growth, tumor progression and the invasiveness of tumor cells [13]. Recently, there have been studies reporting that TROP-2 may be used in the differential diagnosis of follicular epithelial cell derived thyroid lesions $[14,15,16,17]$.

The purpose of this study is to determine the diagnostic value of TROP-2 expression in differential diagnosis of papillary thyroid carcinoma and compare it to the expression of the three most frequently used antibodies (HBME-1, CK19 and Gal-3).

\section{Material and methods}

\section{Patient selection and construction of tissue microarray (TMA) blocks}

From the archive of the Department of Pathology, Recep Tayyip Erdoğan University, Rize, Turkey, 20 normal thyroid (NT) tissue, 23 benign follicular nodular disease (FND), 17 follicular adenoma (FA), 20 follicular variant papillary carcinoma (FVPTC) and 22 classical variant papillary carcinoma (CVPTC) cases were selected. The selection was made by two pathologists (A.R.M. and H.G.) based on the criteria explained below, using a two-headed microscope. The well-delineated nodules surrounded by a thin partial capsule, with a colloidal appearance, without characteristic nuclear features for papillary carcinoma in the thyroidectomy materials belonging to patients who were operated due to multinodular goiter were taken into the FND [18] (also known as nodular hyperplasia or adenomatous hyperplasia) group. Nodules completely surrounded by a thin fibrous capsule, compressing the peripheral thyroid parenchyma, without characteristic nuclear features of papillary carcinoma and without capsular and/or vascular invasion, were included in the FA group. The CVPTC group consisted of cases showing pure papillary growth pattern and characteristic nuclear features of papillary carcinoma. The FVPTC group composed of cases where the tumor was completely sampled, without papilla formation, composed entirely of follicular structures and with characteristic papillary carcinoma nuclear features. In FA, the largest tumor diameter was 6.2 $\mathrm{cm}$, the smallest was $2 \mathrm{~cm}$ and the mean diameter was $3.73 \pm 1.31 \mathrm{~cm}$. In FVPTC, the largest tumor diam- eter was $6 \mathrm{~cm}$, the smallest was $1.1 \mathrm{~cm}$ and the mean diameter was $3.19 \pm 1.50 \mathrm{~cm}$. In CVPTC, the largest tumor diameter was $6 \mathrm{~cm}$, the smallest was 1.3 $\mathrm{cm}$ and the mean diameter was $2.15 \pm 0.98 \mathrm{~cm}$. As this is a TMA-based study, papillary microcarcinoma cases were not included for avoiding loss of materials of patients. Additionally, the non-lesional thyroid parenchyma of the cases operated due to multinodular goiter and not containing any type of thyroiditis were also included in the NT group (Fig. 1). As we did not have any cases with follicular carcinoma in our archive, this group was not included in the study. Slides stained with hematoxylin and eosin (HE) of selected cases were reviewed and the best paraffin blocks for usage in the study were chosen. Two tissue cores representing the lesion were extracted by a dermatological punch biopsy device with $2 \mathrm{~mm}$ in diameter from the selected paraffin blocks. The obtained cores were placed by mapping into the recipient blocks we prepared separately for each group. Approval was obtained from the institutional Research Ethics Board.

\section{Immunohistochemistry}

From each of the constructed TMA blocks, a total of 5 sections with $4 \mu \mathrm{m}$ thickness were taken into positively charged slides. First slide was stained with HE. Other slides were stained on an automated immunohistochemistry system (Roche, Ventana, Benchmark, XT, USA) using the primary antibodies of Mesothelioma (MS-1494, Mouse, monoclonal, clone HBME-1, ready to use, $32 \mathrm{~min}$ incubation, Thermo Scientific, Fremont, CA, USA), CK19 (MS-198, Mouse, monoclonal, clone A53-B/A2.26, ready to use, $32 \mathrm{~min}$ incubation, Thermo Scientific, Fremont, CA, USA), Gal-3 (MS-1756, Mouse, monoclonal, clone 9C4, ready to use, 32 min incubation, Thermo Scientific, Fremont, CA, USA), TROP-2 (Rabbit, polyclonal, 1 : 150 dilution, $15 \mathrm{~min}$ incubation, GeneTex). Signal was visualized with a $3.3^{\prime}$ diaminobenzidine detection kit. All procedures were performed in accordance with the manufacturer's instructions. As positive controls, pleural tissue, skin, normal duodenum and placental tissue were used for HBME-1, CK19, Gal-3 and TROP-2, respectively.

\section{Analysis of immunohistochemical stains}

Immunohistochemical stains were analyzed by two independent pathologists (A.R.M and H.G.) using a two-headed microscope. Membranous staining for HBME-1, membranous \pm cytoplasmic staining for CK19, cytoplasmic \pm nuclear staining for Gal-3, and membranous staining for TROP-2 were accepted as true positive staining.

In the immunohistochemical markers in cases, the staining intensity was analyzed in four categories as negative (0), weak (1), moderate (2) and strong (3), 

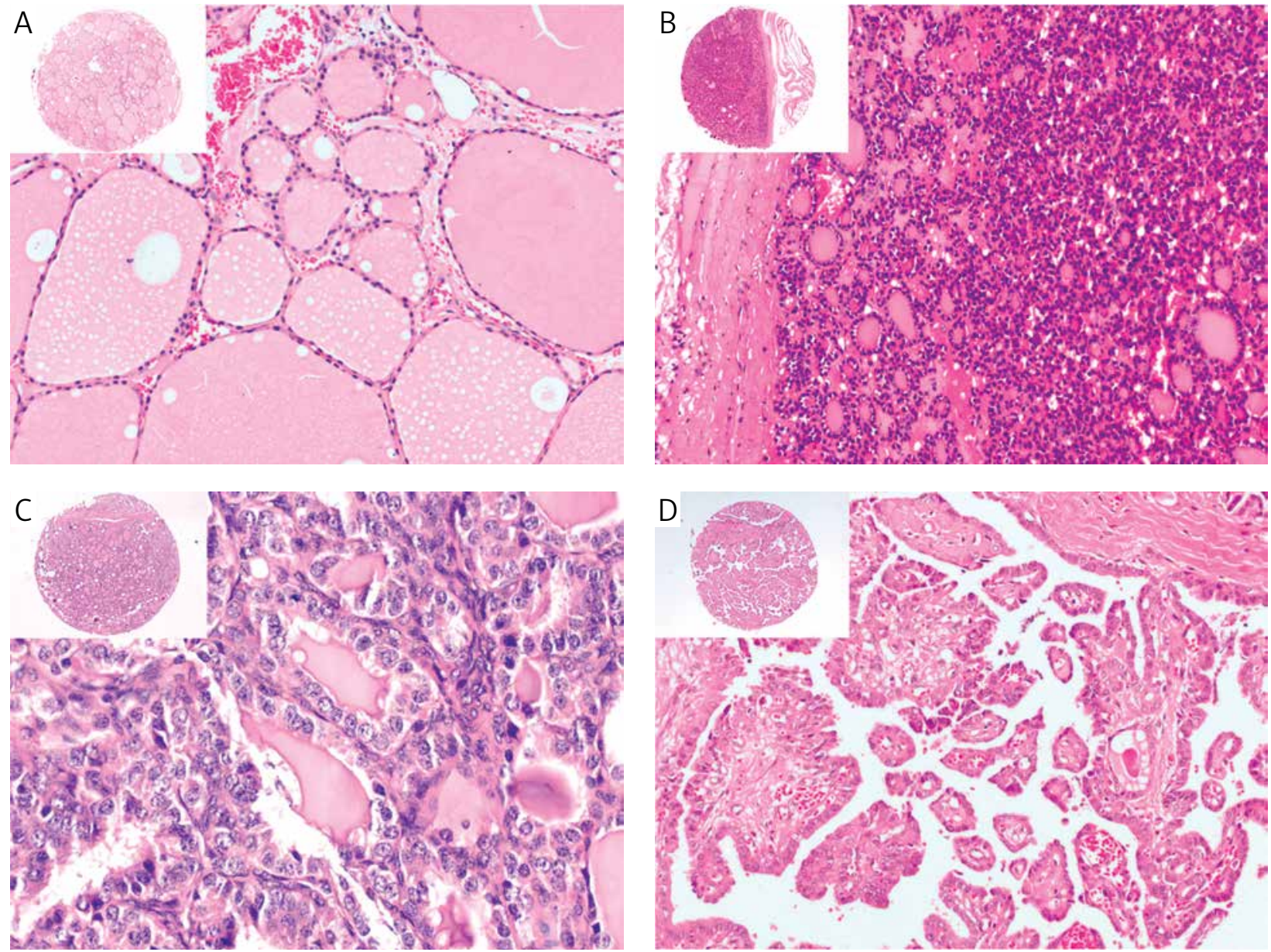

Fig. 1. A) Follicular nodular disease (HE, original magnification 40-200×). B) Follicular adenoma (HE, original magnification 40-200×). C) Follicular variant papillary carcinoma (HE, original magnification 40-200×). D) Classical variant papillary carcinoma (HE, original magnification 40-200×)

and the staining percentage was analyzed in five categories as $<1 \%(0), 1-25 \%(1), 26-50 \%(2), 51-75 \%$ (3) and $76-100 \%$ (4). A total expression score (TES) was calculated for each case (Table I). Cases with a TES of 1 or higher were accepted as positive.

\section{Statistical analysis}

Statistical Package for social sciences (SPSS) 23.0 was used to statistically analyze the obtained findings. While analyzing the data in the study, in addition to descriptive statistical methods (mean, standard deviation), Mann Whitney $\mathrm{U}$ test was used to compare quantitative data. $\chi^{2}$ test and Fischer's Exact test were used in comparing the qualitative data. $\mathrm{p}$ values less than 0.05 were considered statistically significant.

\section{Results}

Expression of immunohistochemical markers in case groups

All of the cases in NT, FND and FA were negative with HBME-1, Gal-3 and TROP-2. On the other hand, CK19 was positive for all NT (100\%), $10 \mathrm{FND}$ $(43.5 \%)$ and 6 FA $(35.3 \%)$ cases. While all cases in CVPTC had positive immunoreaction with HBME-1 and Gal-3, CK19 was positive in $95.5 \%(21 / 22)$, TROP-2 was positive in $90.9 \%$ (20/22). In FVPTC,

Table I. Total expression scores of immunohistochemical markers in terms of staining intensity and percentage

\begin{tabular}{cc}
\hline TES & $($ SI, SP) \\
\hline 0 & $(0,0)(1,0)(0,1)$ \\
\hline 1 & $(1,1)$ \\
\hline 2 & $(1,2)(2,1)$ \\
\hline 3 & $(1,3)(3,1)$ \\
\hline 4 & $(1,4)(2,2)$ \\
\hline 5 & $(2,3)(3,2)$ \\
\hline 6 & $(2,4)$ \\
\hline 7 & $(3,3)$ \\
\hline 8 & $(3,4)$ \\
\hline
\end{tabular}

TES - total expression score; SI - staining intensity; SP - staining percentage 
14 cases (70\%) were positive with CK19, 9 (45\%) were positive with HBME-1, and 7 (35) were positive with Gal-3, while TROP-2 was positive in only $1(5 \%)$ case (Figs. 2, 3). Table II shows the expression of immunohistochemical markers in case groups.

\section{Comparison of TES means of immunohistochemical markers}

The antibody with the highest mean TES was Gal-3 in CVPTC (8.00 \pm 0.00$)$ and CK19 in FVPTC $(3.75 \pm 3.19)$. TROP-2 had the lowest mean TES in both groups $(5.14 \pm 3.06$ and $0.20 \pm 0.89)$. CK19's mean TES was $4.05 \pm 1.93$ in NT, $0.87 \pm 1.29$ in FND and $1.00 \pm 1.70$ in FA. These values were 0 for the other three antibodies. Table III shows the mean TES values and standard deviations of immunohistochemical markers in case groups.

For all markers, TES means in groups with papillary carcinoma and classic variant papillary carcinoma were higher than those with benign thyroid lesions in FND and FA ( $p<0.001)$. The mean TES of the CVPTC group was found significantly higher than that of the FVPTC group $(\mathrm{p}<0.001)$. The mean TES of HBME-1, Gal-3 and CK19 in the FVPTC group was found higher than that of the group with FND + FA $(\mathrm{p}<0.001$ and $\mathrm{p}=0.001)$. We did not find a statistically significant difference in terms of TROP-2 TES means between benign thyroid lesions and the FVPTC group ( $=0.157$ ). The $p$ values showing the relationship between case groups and immunohistochemical markers are shown in Table IV.

The diagnostic value of immunohistochemical markers in distinguishing between malignant lesions and benign lesions

HBME-1, CK19, Gal-3 and TROP-2 were found statistically significant in distinguishing between benign lesions and papillary carcinomas $(\mathrm{p}<0.001)$. If positivity of one antibody is accepted for diagnosis of papillary carcinoma, the
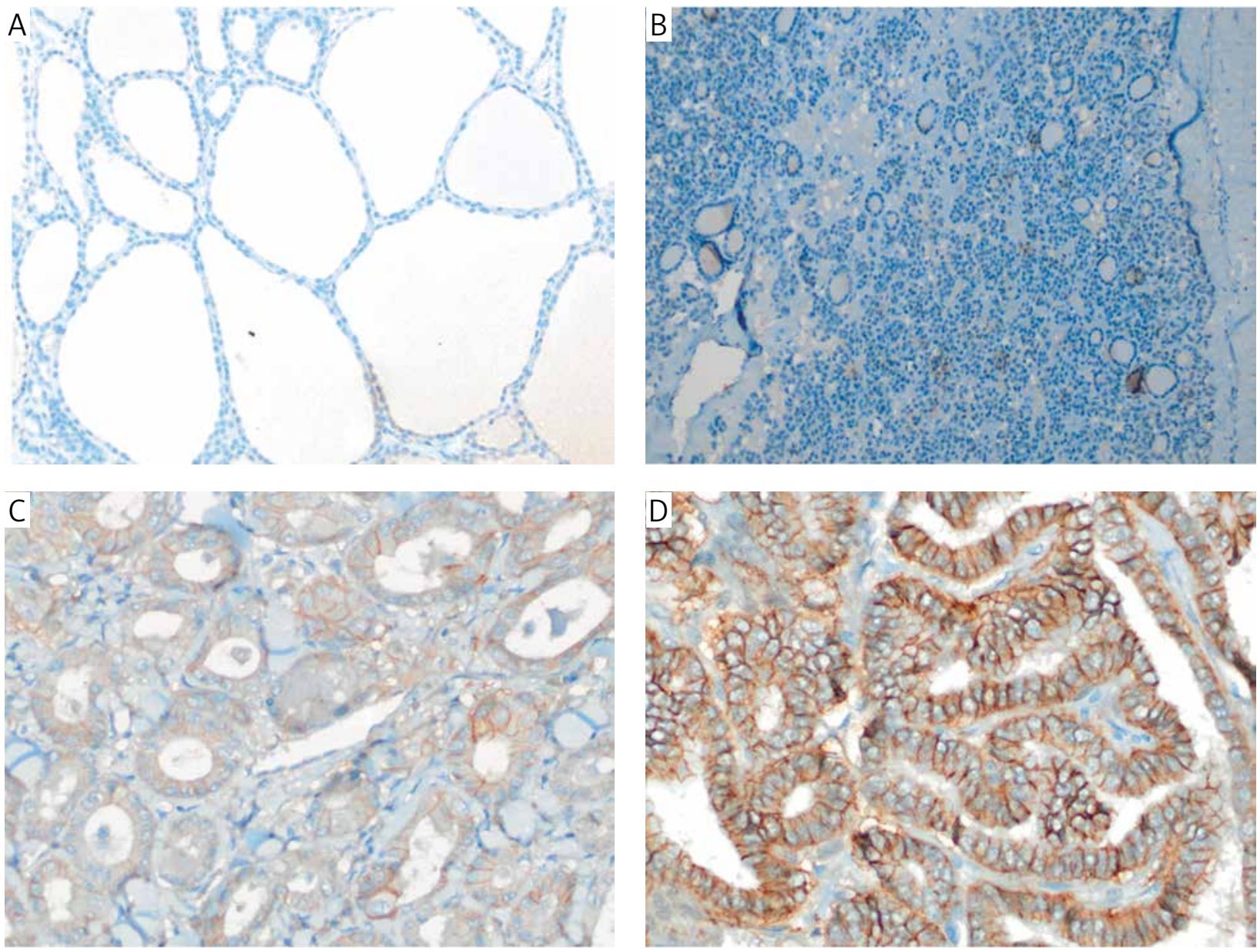

Fig. 2. A) Negative TROP2 expression in a case of follicular nodular disease (original magnification $100 \times$ ). B) Negative TROP2 expression in a case of follicular adenoma (original magnification $100 \times$ ). C) Focal moderate membranous immunostaining of TROP2 in a case of follicular variant papillary carcinoma (original magnification $100 \times$ ). D) Diffuse and strong membranous immunostaining of TROP2 in a case of classical variant papillary carcinoma (original magnification $100 \times)$ 

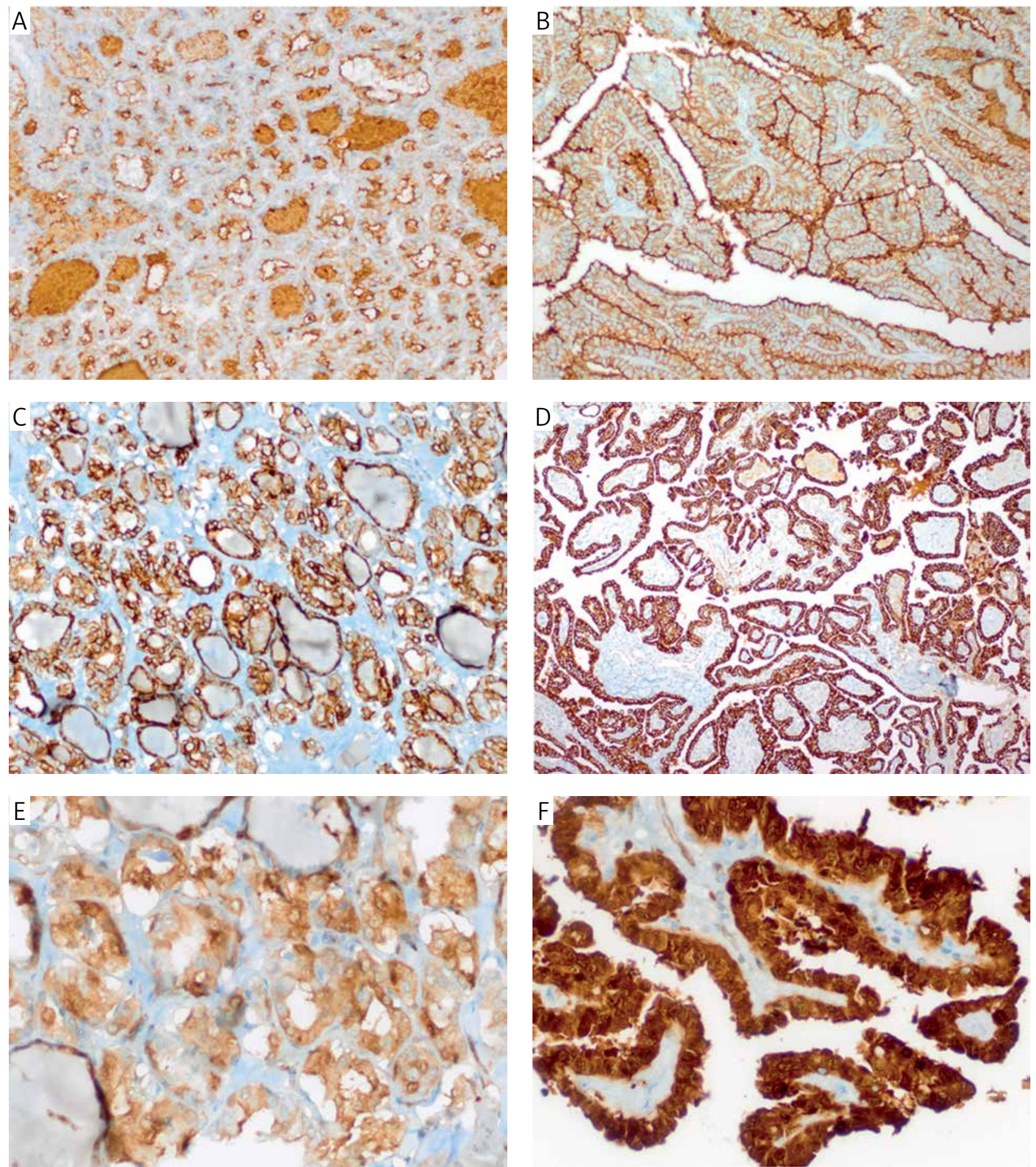

Fig. 3. A) Apical and membranous HBME-1 positivity in a case of follicular variant papillary carcinoma (original magnification $100 \times$ ). B) Strong and diffuse membranous HBME-1 positivity in a case of classical variant papillary carcinoma (original magnification $100 \times$ ). C, D) Cytoplasmic and membranous CK19 positivity in cases of FVPTC and CVPTC (original magnification $100 \times$ ). E) Galectin 3 positivity in a case of FVPTC (original magnification $100 \times$ ). F) Strong cytoplasmic and nuclear galectin 3 positivity in a case of CVPTC (original magnification $100 \times$ )

usage of Gal-3, TROP-2 and CK19 together, with or without HBME-1 had the highest sensitivity $(90.5 \%)$. However, the specificity of these combinations was $60 \%$ due to the positivity of CK19 in benign lesions. When CK19 was kept out, specificity of the combination of other antibodies reach $100 \%$. In this case, the combination of HBME-1,
Gal-3 and TROP-2 (78.6-89\%) had the highest sensitivity and diagnostic accuracy. In differential diagnoses between benign lesions (FND + FA) and papillary carcinoma (FVPTC + CVPTC), the sensitivity, specificity, positive predictive value ( $\mathrm{PPV})$, negative predictive value (NPV) and diagnostic accuracy (DA) determined for antibodies are shown in Table V. 
Table II. The expression of immunohistochemical markers in case groups

\begin{tabular}{|c|c|c|c|c|c|c|c|c|}
\hline $\begin{array}{l}\text { IMMUNOHIS- } \\
\text { TOCHEMICAL } \\
\text { MARKERS }\end{array}$ & & $\begin{array}{c}\text { NT } \\
\text { N }(\%)\end{array}$ & $\begin{array}{l}\text { FND } \\
\text { N }(\%)\end{array}$ & $\begin{array}{c}\text { FA } \\
\mathrm{N}(\%)\end{array}$ & $\begin{array}{c}\text { FND + FA } \\
\text { N }(\%)\end{array}$ & $\begin{array}{l}\text { FVPTC } \\
\text { N }(\%)\end{array}$ & $\begin{array}{l}\text { CVPTC } \\
\text { N }(\%)\end{array}$ & $\begin{array}{c}\text { FVPTC + } \\
\text { CVPTC } \\
\text { N }(\%)\end{array}$ \\
\hline \multirow[t]{2}{*}{ HBME-1 } & + & 0 & 0 & 0 & 0 & $9(45)$ & $22(100)$ & $31(73.8)$ \\
\hline & - & $20(100)$ & $23(100)$ & $17(100)$ & $40(100)$ & $11(55)$ & 0 & $11(26.2)$ \\
\hline \multirow[t]{2}{*}{ CK19 } & + & $20(100)$ & $10(43.5)$ & $6(35.3)$ & $16(40)$ & $14(70)$ & $21(95.5)$ & $35(83.3)$ \\
\hline & - & 0 & $13(56.5)$ & $11(64.7)$ & $24(60)$ & $6(30)$ & $1(4.5)$ & $7(16.7)$ \\
\hline \multirow[t]{2}{*}{ Gal-3 } & + & 0 & 0 & 0 & 0 & $7(35)$ & $22(100)$ & 29 (69) \\
\hline & - & $20(100)$ & $23(100)$ & $17(100)$ & $40(100)$ & $13(65)$ & 0 & $13(31)$ \\
\hline \multirow[t]{2}{*}{ TROP-2 } & + & 0 & 0 & 0 & 0 & $1(5)$ & 20 (90.9) & $21(50)$ \\
\hline & - & $20(100)$ & $23(100)$ & $17(100)$ & $40(100)$ & $19(95)$ & $2(9.1)$ & $21(50)$ \\
\hline
\end{tabular}

NT - normal thyroid; FND - follicular nodular disease; FA - follicular adenoma; FVPTC - follicular variant papillary carcinoma; CVPTC - classical variant papillary carcinoma

Table III. The mean total expression scores and standard deviations of immunohistochemical markers in groups

\begin{tabular}{lccccccc}
\hline $\begin{array}{l}\text { IMMUNOHIS- } \\
\text { TOCHEMICAL } \\
\text { MARKERS }\end{array}$ & NT & FND & FA & FND + FA & FVPTC & CVPTC & $\begin{array}{c}\text { FVPTC + } \\
\text { CVPTC }\end{array}$ \\
\hline HBME-1 & $0.00 \pm 0.00$ & $0.00 \pm 0.00$ & $0.00 \pm 0.00$ & $0.00 \pm 0.00$ & $2.70 \pm 3.31$ & $6.68 \pm 1.62$ & $4.79 \pm 3.23$ \\
\hline CK19 & $4.05 \pm 1.93$ & $0.87 \pm 1.29$ & $1.00 \pm 1.70$ & $0.93 \pm 1.46$ & $3.75 \pm 3.19$ & $7.36 \pm 1.89$ & $5.64 \pm 3.15$ \\
\hline Gal-3 & $0.00 \pm 0.00$ & $0.00 \pm 0.00$ & $0.00 \pm 0.00$ & $0.00 \pm 0.00$ & $1.30 \pm 2.16$ & $8.00 \pm 0.00$ & $4.81 \pm 3.69$ \\
\hline TROP-2 & $0.00 \pm 0.00$ & $0.00 \pm 0.00$ & $0.00 \pm 0.00$ & $0.00 \pm 0.00$ & $0.20 \pm 0.89$ & $5.14 \pm 3.06$ & $2.79 \pm 3.38$ \\
\hline
\end{tabular}

NT - normal thyroid; FND - follicular nodular disease; FA - follicular adenoma; FVPTC - follicular variant papillary carcinoma; CVPTC - classical variant papillary carcinoma

Table IV. P values demonstrating the relationship between groups and the mean TES values of the immunohistochemical markers

\begin{tabular}{lcccc}
\hline $\begin{array}{l}\text { IMMUNOHISTOCHEMICAL } \\
\text { MARKERS }\end{array}$ & $\begin{array}{c}\text { FND + FA / } \\
\text { FVPTC + CVPTC }\end{array}$ & $\begin{array}{c}\text { FND + FA / } \\
\text { FVPTC }\end{array}$ & $\begin{array}{c}\text { FND + FA / } \\
\text { CVPTC }\end{array}$ & $\begin{array}{c}\text { FVPTC/ } \\
\text { CVPTC }\end{array}$ \\
\hline HBME-1 & $\mathrm{p}<0.001$ & $\mathrm{p}<0.001$ & $\mathrm{p}<0.001$ & $\mathrm{p}<0.001$ \\
\hline CK19 & $\mathrm{p}<0.001$ & $\mathrm{p}=0.001$ & $\mathrm{p}<0.001$ & $\mathrm{p}<0.001$ \\
\hline Gal-3 & $\mathrm{p}<0.001$ & $\mathrm{p}<0.001$ & $\mathrm{p}<0.001$ & $\mathrm{p}<0.001$ \\
\hline TROP-2 & $\mathrm{p}<0.001$ & $\mathrm{p}=0.157$ & $\mathrm{p}<0.001$ & $\mathrm{p}<0.001$ \\
\hline
\end{tabular}

$F N D$ - follicular nodular disease; $F A$ - follicular adenoma; FVPTC - follicular variant papillary carcinoma; CVPTC - classical variant papillary carcinoma

The diagnostic value of immunohistochemical markers in distinguishing follicular variant papillary carcinoma from benign lesions

While HBME-1 and Gal-3 were statistically significant in distinguishing benign lesions from FVPTCs $(\mathrm{p}<0.001)$, CK19 and TROP-2 were not significant in distinguishing these two $(\mathrm{p}=0.055$ and $\mathrm{p}=$ 0.333 ). In distinguishing diagnoses of benign lesions (FND + FA) from FVPTCs, the sensitivity, specificity, positive predictive value (PPV), negative predictive value (NPV) and diagnostic accuracy (DA) determined for antibodies are shown in Table VI.

\section{Discussion}

In combination of optimal tissue processing and slide quality by an experienced pathologist, most thyroid lesions, especially CVPTC, may be diagnosed without needing an additional ancillary technique. However, it is known that there are disagreements among even endocrine pathologists analyzing the same lesion, in suspicious thyroid nodules [2]. Factors that affect the variety among observers include education of pathologists in different centers and by different educators [19]. As far as we observed in consultations made to our department, even distinguish- 
Table V. Sensitivity, specificity, positive predictive value (PPV), negative predictive value (NPV) and diagnostic accuracy (DA) in benign lesions (FND + FA) against papillary carcinoma (FVPTC + CVPTC) for any immunohistochemical marker or their combinations

\begin{tabular}{|c|c|c|c|c|c|}
\hline IMMUNOHISTOCHEMICAL MARKERS & SENSITIVITY $(\%)$ & SPECIFICITY $(\%)$ & PPV $(\%)$ & NPV (\%) & $\mathrm{DA}(\%)$ \\
\hline HBME-1 & 73.8 & 100 & 100 & 78.4 & 87 \\
\hline CK19 & 83.3 & 60 & 68.6 & 77.4 & 72 \\
\hline Gal-3 & 69 & 100 & 100 & 75.5 & 84 \\
\hline TROP-2 & 50 & 100 & 100 & 65.6 & 74 \\
\hline HBME-1 + CK19 & 85.7 & 60 & 69.2 & 80 & 73 \\
\hline HBME-1 + Gal-3 & 76.2 & 100 & 100 & 80 & 89 \\
\hline $\mathrm{CK} 19+\mathrm{Gal}-3$ & 88.1 & 60 & 69.8 & 82.8 & 74 \\
\hline HBME-1 + CK19 + Gal-3 & 88.1 & 60 & 69.8 & 82.8 & 74 \\
\hline TROP-2 + HBME-1 & 76.2 & 100 & 100 & 80 & 89 \\
\hline TROP-2 + CK19 & 85.7 & 60 & 69.2 & 80 & 73 \\
\hline TROP-2 + Gal-3 & 71.4 & 100 & 100 & 76.9 & 85 \\
\hline TROP-2 + HBME-1 + CK19 & 88.1 & 60 & 69.8 & 82.8 & 74 \\
\hline TROP-2 + HBME-1 + Gal-3 & 78.6 & 100 & 100 & 81.6 & 89 \\
\hline TROP-2 + CK19 + Gal-3 & 90.5 & 60 & 70.4 & 85.7 & 76 \\
\hline $\begin{array}{l}\text { HBME-1 + CK19+ Gal-3 + } \\
\text { TROP-2 }\end{array}$ & 90.5 & 60 & 70.4 & 85.7 & 76 \\
\hline
\end{tabular}

Table VI. Sensitivity, specificity, positive predictive value (PPV), negative predictive value (NPV) and diagnostic accuracy (DA) in benign lesions (FND + FA) against FVPTC for any immunohistochemical marker

\begin{tabular}{lccccc}
\hline IMMUNOHISTOCHEMICAL MARKERS & SENSITIVITY $(\%)$ & SPECIFICITY $(\%)$ & PPV (\%) & NPV (\%) & DA (\%) \\
\hline HBME-1 & 45 & 100 & 100 & 78.4 & 81 \\
\hline CK19 & 70 & 60 & 46.7 & 80 & 63 \\
\hline Gal-3 & 35 & 100 & 100 & 75.5 & 78 \\
\hline TROP-2 & 5 & 100 & 100 & 67.8 & 68 \\
\hline
\end{tabular}

ing benign thyroid lesions with papillary growth pattern (hyperplastic nodule, hyperfunctioning adenoma, Graves' disease, etc.) and CVPTCs may be hard for inexperienced pathologists. In our study, we compared the diagnostic values of HBME-1, CK19 and Gal-3, which are used frequently in distinguishing papillary carcinoma from benign thyroid lesions, and TROP-2, which started to be studied recently.

CK19 is a low molecular weight cytokeratin which is expressed in various epithelial tissues and tumors [10]. While there are differences among studies in terms of the extensiveness and intensity of its expression in follicular epithelial cell derived lesions of the thyroid, it is more diffused and stronger in papillary carcinoma $[5,8,9,10]$. Additionally, it is known to be positively stained also in benign nodules and follicular adenoma $[7,8]$. In Cheung et al.'s study, $80 \%$ of CVPTCs, $57 \%$ of FVPTCs, and $20 \%$ of nodular hyperplasia and FAs were positive with CK19 [5]. In our study CK19 was positively stained in respectively 95.5\% and $70 \%$ of cases with CVPTC and FVPTC, while it was positively stained in respectively $100 \%$, $43.5 \%$ and $35.5 \%$ of normal thyroid tissue, follicular nodular disease and follicular adenoma. The mean TES was lower in FVPTC than CVPTC. While the mean TES was 0.87 and 1.00 in FND and FA respectively, it was significantly lower than those in the FVPTC and CVPTC groups. In Dunderovic et al.'s literature review, the researchers found the sensitivity and specificity of CK19 in distinguishing malignant and benign lesions as $80 \%$ and $78 \%$ respectively. These were respectively $74 \%$ and $77 \%$ in distinguishing FVPTC and FA [12]. In our study, these ratios were respectively $83.3 \%$ and $60 \%$ for distinguishing FND-FA and PTC, and $70 \%$ and $60 \%$ for distinguishing FND-FA and FVPTC. In agreement 
with the literature $[4,8]$, we observed that usage of CK19 by itself had the highest sensitivity as a marker in distinguishing benign lesions from PTC and FVPTC $(83.3 \%, 70 \%)$. However, its specificity was lower due to its positivity in benign lesions $(60 \%)$.

Interestingly, we observed a stronger and more extensive staining with CK19 in normal thyroid tissues in comparison to FND and FA cases. This finding would not create an issue to easily recognize normal thyroid tissue during microscopic examination of the thyroidectomy material. However, it may cause misdiagnosis in cell block materials collected from normal thyroid tissues instead of lesion. For cytological material, we think applying diagnostic histopathological criteria solidly, and adding other ancillary markers to the panel will help overcome this problem.

HBME-1 is a monoclonal antibody developed against the antigen on the microvillous surface of human mesothelial cells [20]. There are numerous studies reporting its advantages in determining malignancy in thyroid nodules derived from follicular epithelial cells $[5,8,10,21]$. Moreover, there are also studies showing HBME- 1 as positive in benign thyroid lesions such as chronic lymphocytic thyroiditis, benign thyroid nodules and follicular adenoma [7, 9, 22]. While Cheung et al. did not find positivity with HBME-1 in any of the benign thyroid nodules and cases of follicular adenoma, they found positivity in $55 \%$ of cases with papillary carcinoma, $70 \%$ in CVPTC, and only $45 \%$ in FVPTC [5]. In Mataraci et al.'s study, all of the 40 hyperplastic thyroid nodules and 35 follicular adenomas were negative with HBME-1 [10]. Similarly, in our study, we did not observe positivity with HBME-1 in benign thyroid nodules and follicular adenoma. While HBME-1 showed a positive expression in all CVPTC cases, this ratio was much lower in FVPTC (45\%). Despite this low ratio observed for FVPTC, the second most sensitive marker in distinguishing benign lesions from PTC and FVPTC was HBME1. As opposed to the findings of de Matos et al. [9], we found that the TES of HBME-1 was also able to distinguish between the group CVPTC and FVPTC $(\mathrm{p}<0.001)$.

Gal-3, chimera type galectin, is a beta galactoside-binding animal lectin known to be related to various mechanisms in tumor development. It has a cytoplasmic and nuclear distribution [23]. It is also stained in reactive epithelial cells and inflammatory cells $\{22\}$. There are many studies demonstrating the positivity of Gal-3 in malignant lesions of thyroid, especially CVPTC $[7,8,9,10,22]$. Furthermore, positivity with varying frequencies was reported in different studies in benign thyroid nodules and lesions, follicular adenoma and FVPTC $[7,8,9,10$, $12,22]$. In our study, we did not observe staining with Gal-3 in NT tissue, and in FND and FA. 69\% of PTCs had staining with Gal-3. We observed staining in $100 \%$ of cases with CVPTC and $35 \%$ of cases with FVPTC ( $\mathrm{p}<0.001)$. In the study by de Matos et al., as in our study, no staining was found in normal thyroid tissue [9]. Various studies demonstrated that Gal-3, in comparison to HBME-1 and CK19, has a higher sensitivity in distinguishing between benign and malignant lesions [7, 12]. In our study, the sensitivity value of Gal-3 was lower than those of HBME-1 and CK19. Its specificity was comparable to that of HBME-1 (100\%). The mean TES for Gal-3 was higher in malignant cases than benign cases, and in CVPTC cases than FVPTC cases ( $\mathrm{p}<0.001)$. Mataraci et al., as found in our study, found the Gal-3 staining percentages and intensity in benign lesions much lower than those in malignant lesions [10]. Matos et al. observed diffuse staining in FVPTC, focal and weak positivity in FA and adenomatous nodules with Gal-3 [9]. As in found our study, they reported that Gal-3 was useful in distinguishing FVPTC cases from benign lesions.

TROP-2, which is a transmembrane glycoprotein coded by the Tacstd 2 gene, is expressed in various normal tissues, while it is over-expressed in larynx, lung, colon, breast, pancreas and gall bladder cancers $[13,24,25,26,27,28,29,30]$. Over-expression of TROP-2, which is accepted as an oncogene, is known as a poor prognostic indicator for various types of cancer, and makes TROP-2 a target molecule in treatment [13]. Furthermore, reduction was observed in the invasiveness of tumor cells by usage of antibodies developed against TROP-2 in breast and colon cancer $[31,32]$.

Over-expression of TROP-2 in various cancers also paved the way to its investigation in thyroid lesions. Studies, except one, demonstrated that TROP-2 was negative in neoplastic and non-neoplastic benign thyroid tissues [15, 16, 17]. Addati et al. reported TROP- 2 positivity in 4 non-neoplastic thyroid tissues and 2 cases of follicular adenoma [14]. In our study, we did not detect positivity with TROP-2 in normal thyroid tissue, in FND or in FA.

TROP-2 positivity was found by Simms et al. in $90 \%(54 / 60)$ in CVPTC and $18.8 \%(9 / 48)$ in FVPTC, while it was found by Liu et al. in $94 \%$ $(33 / 35)$ in CVPTC and 81\% (30/37) in FVPTC [16, 17]. Bychkov et al., as opposed to what they observed in CVPTC, found TROP-2 positivity in less than half of the FVPTC cases. Most of these were showing focal positivity. A small number of FVPTC showed diffuse staining [15]. In similarity to other studies, TROP-2 was positive in $90.9 \%$ of our cases with CVPTC. Our TROP-2-positive number of FVPTC cases was fewer than those in all other studies. We now know as a result of molecular studies that, as opposed to CVPTC which contains mutations such as $B R A F$ V600E, FA, follicular thyroid carcinoma and 
encapsulated FVPTC contain $R A S$ and similar mutations [33]. According to some studies, on the other hand, tumors containing few papillary structures and showing dominant follicular growth patterns, contain $B R A F$ V600E mutation, and these should be accepted as CVPTC $[34,35]$. However, recognition of papillary structures is subjective even among experienced endocrine pathologists. In a study proposing the terminology "Noninvasive follicular thyroid neoplasm with papillary-like nuclear features" (NIFTP), after review, 17 of the 130 cases diagnosed with FVPTC with invasion were excluded from the study as they had papillary structures of higher than $1 \%$ [3]. Higher rates of TROP-2 positivity in FVPTC shown in other studies may have been caused by not having sampled the lesions completely and/or the researchers missing the papillary structures in the lesion. These cases which have a different molecular pathway and should be defined as CVPTC included in the FVPTC group may have caused different results. In our study, although we sampled lesions in toto and excluded all cases involving papilla from the group, we found focal TROP-2 positivity of moderate intensity in $1(5 \%)$ of the cases with FVPTC. The TROP-2 over-expression in CVPTC, most of which contains $B R A F \mathrm{~V} 600 \mathrm{E}$ mutation, suggests that this molecule known to activate the MAPK (ERK1/2) pathway is related to CVPTC carcinogenesis. In order to reveal the relationship between $B R A F \mathrm{~V} 600 \mathrm{E}$ mutation and TROP-2 over-expression, research should be collected on crowded series of cases with certainty of $B R A F$-like phenotypes. Another possible reason for high percentage of negativity may be the tumor heterogeneity observed in TMA studies with a lower volume of tissues represented.

In conclusion, microscopic examinations performed with conventional staining procedures still protect their value. In distinguishing papillary carcinoma from benign thyroid nodules including follicular adenoma, it may be a necessity to use ancillary techniques, especially immunohistochemical markers. While our study was composed of a small number of cases, it showed that; while the negativity of any marker by itself in a suspicious thyroid nodule does not exclude malignancy, positivity in any of the HBME-1, Gal-3 or TROP-2 markers supports the diagnosis of papillary carcinoma. It should also be remembered that CK19 may be focally and weakly stained in benign thyroid nodules. By all these reasons, in distinguishing papillary carcinoma from benign lesions, we recommend the usage of HBME-1, CK19, Gal-3 and TROP-2 as panel.

The authors would like to thank to Busra Demircan for her excellent technical assistance.

The authors declare no conflict of interest.

Financial disclosure: None

\section{References}

1. LiVolsi VA. Papillary thyroid carcinoma: an update. Mod Pathol 2011; 24 Suppl 2: S1-9.

2. Elsheikh TM, Asa SL, Chan JK, et al. Interobserver and intraobserver variation among experts in the diagnosis of thyroid follicular lesions with borderline nuclear features of papillary carcinoma. Am J Clin Pathol 2008; 130: 736-744.

3. Nikiforov YE, Seethala RR, Tallini G, et al. Nomenclature revision for encapsulated follicular variant of papillary thyroid carcinoma: a paradigm shift to reduce overtreatment of indolent tumors. JAMA Oncol. 2016; 2: 1023-1029.

4. Ma H, Xu S, Yan J, et al. The value of tumor markers in the diagnosis of papillary thyroid carcinoma alone and in combination. Pol J Pathol 2014; 65: 202-209.

5. Cheung CC, Ezzat S, Freeman JL, et al. Immunohistochemical diagnosis of papillary thyroid carcinoma. Mod Pathol. 2001; 14: 338-342.

6. Nechifor-Boila A, Catana R, Loghin A, et al. Diagnostic value of HBME-1, CD56, Galectin-3 and Cytokeratin-19 in papillary thyroid carcinomas and thyroid tumors of uncertain malignant potential. Rom J Morphol Embryol 2014; 55: $49-56$.

7. Park YJ, Kwak SH, Kim DC, et al. Diagnostic value of galectin-3, HBME-1, cytokeratin 19, high molecular weight cytokeratin, cyclin D1 and p27(kip1) in the differential diagnosis of thyroid nodules. J Korean Med Sci 2007; 22: 621-628.

8. Barut F, Onak Kandemir N, Bektas S, et al. Universal markers of thyroid malignancies: galectin-3, HBME-1, and cytokeratin-19. Endocr Pathol 2010; 21: 80-89.

9. de Matos PS, Ferreira AP, de Oliveira Facuri F, et al. Usefulness of HBME-1, cytokeratin 19 and galectin-3 immunostaining in the diagnosis of thyroid malignancy. Histopathology 2005; 47: 391-401.

10. Mataraci EA, Ozguven BY, Kabukcuoglu F. Expression of cytokeratin 19, HBME-1 and galectin-3 in neoplastic and nonneoplastic thyroid lesions. Pol J Pathol 2012; 63: 58-64.

11. Ozolins A, Narbuts Z, Strumfa I, et al. Immunohistochemical expression of HBME-1, E-cadherin, and CD56 in the differential diagnosis of thyroid nodules. Medicina (Kaunas) 2012; 48: 507-514.

12. Dunderovic D, Lipkovski JM, Boricic I, et al. Defining the value of CD56, CK19, Galectin 3 and HBME-1 in diagnosis of follicular cell derived lesions of thyroid with systematic review of literature. Diagn Pathol. 2015; 10: 196.

13. Shvartsur A, Bonavida B. Trop2 and its overexpression in cancers: regulation and clinical/therapeutic implications. Genes Cancer 2015; 6: 84-105.

14. Addati T, Achille G, Centrone M, et al. TROP-2 expression in papillary thyroid cancer: a preliminary cyto-histological study. Cytopathology 2015; 26: 303-311.

15. Bychkov A, Sampatanukul P, Shuangshoti S, et al. TROP-2 immunohistochemistry: a highly accurate method in the differential diagnosis of papillary thyroid carcinoma. Pathology. 2016; 48: 425-433.

16. Liu H, Shi J, Lin F. The Potential Diagnostic Utility of TROP-2 in Thyroid Neoplasms. Appl Immunohistochem Mol Morphol 2016.

17. Simms A, Jacob RP, Cohen C, et al. TROP-2 expression in papillary thyroid carcinoma: potential diagnostic utility. Diagn Cytopathol 2016; 44: 26-31.

18. Mete O, Asa SL. Pitfalls in the diagnosis of follicular epithelial proliferations of the thyroid. Adv Anat Pathol 2012; 19: 363373.

19. Hirokawa M, Carney JA, Goellner JR, et al. Observer variation of encapsulated follicular lesions of the thyroid gland. Am J Surg Pathol. 2002; 26: 1508-1514.

20. Sack MJ, Astengo-Osuna C, Lin BT, et al. HBME-1 immunostaining in thyroid fine-needle aspirations: a useful marker in the diagnosis of carcinoma. Mod Pathol 1997; 10: 668-674. 
21. Gucer H, Bagci P, Bedir R, et al. The value of HBME-1 and claudin-1 expression profile in the distinction of BRAF-like and RAS-like phenotypes in papillary thyroid carcinoma. Endocr Pathol 2016; 27: 224-232.

22. Scognamiglio T, Hyjek E, Kao J, et al. Diagnostic usefulness of HBME1, galectin-3, CK19, and CITED1 and evaluation of their expression in encapsulated lesions with questionable features of papillary thyroid carcinoma. Am J Clin Pathol. 2006; 126: 700-708.

23. Dondoo TO, Fukumori T, Daizumoto K, et al. Galectin-3 is implicated in tumor progression and resistance to anti-androgen drug through regulation of androgen receptor signaling in prostate cancer. Anticancer Res 2017; 37: 125-134.

24. Chen MB, Wu HF, Zhan Y, et al. Prognostic value of TROP2 expression in patients with gallbladder cancer. Tumour Biol 2014; 35: 11565-11569.

25. Chen R, Lu M, Wang J, et al. Increased expression of Trop2 correlates with poor survival in extranodal NK/T cell lymphoma, nasal type. Virchows Arch 2013; 463: 713-719.

26. Fang YJ, Lu ZH, Wang GQ, et al. Elevated expressions of MMP7, TROP2, and survivin are associated with survival, disease recurrence, and liver metastasis of colon cancer. Int J Colorectal Dis 2009; 24: 875-884.

27. Fong D, Moser P, Krammel C, et al. High expression of TROP2 correlates with poor prognosis in pancreatic cancer. $\mathrm{Br} \mathrm{J}$ Cancer 2008; 99: 1290-1295.

28. Muhlmann G, Spizzo G, Gostner J, et al. TROP2 expression as prognostic marker for gastric carcinoma. J Clin Pathol 2009; 62: 152-158.

29. Ning S, Liang N, Liu B, et al. TROP2 expression and its correlation with tumor proliferation and angiogenesis in human gliomas. Neurol Sci 2013; 34: 1745-1750.

30. Wu H, Xu H, Zhang S, et al. Potential therapeutic target and independent prognostic marker of TROP2 in laryngeal squamous cell carcinoma. Head Neck 2013; 35: 1373-1378.

31. Lin $\mathrm{H}$, Zhang $\mathrm{H}$, Wang J, et al. A novel human Fab antibody for Trop2 inhibits breast cancer growth in vitro and in vivo. Int J Cancer 2014; 134: 1239-1249.

32. Wang J, Day R, Dong Y, et al. Identification of Trop-2 as an oncogene and an attractive therapeutic target in colon cancers. Mol Cancer Ther 2008; 7: 280-285.

33. Agrawal N, Akbani R, Aksoy BA, et al. Integrated genomic characterization of papillary thyroid carcinoma. Cell 2014; 159: 676-690.

34. Jakubowski M, Hunt JL. BRAF mutational analysis in papillary carcinomas with mixed follicular and papillary growth patterns. Am J Surg Pathol. 2009; 33: 1590-1593.

35. Onder S, Ozturk Sari S, Yegen G, et al. Classic Architecture with multicentricity and local recurrence, and absence of TERT promoter mutations are correlates of BRAF (V600E) harboring pediatric papillary thyroid carcinomas. Endocr Pathol 2016; 27: 153-161.

\section{Address for correspondence}

Afsin Rahman Murtezaoglu

Department of Pathology

Recep Tayyip Erdogan University

Rize, Turkey

e-mail: afsinmurtezaoglu@hotmail.com 